\title{
Phenotypical and Genotypical Properties of an Arcanobacterium pluranimalium Strain Isolated from a Juvenile Giraffe (Giraffa camelopardalis reticulata)
}

\author{
Karin Risse, ${ }^{1}$ Karen Schlez, ${ }^{1}$ Tobias Eisenberg, ${ }^{1}$ Christina Geiger, ${ }^{2}$ Anna Balbutskaya, ${ }^{3}$ \\ Osama Sammra, ${ }^{3}$ Christoph Lämmler, ${ }^{3}$ and Amir Abdulmawjood ${ }^{4}$ \\ ${ }^{1}$ Landesbetrieb Hessisches Landeslabor, Schubertstraße 60, 35392 Gießen, Germany \\ ${ }^{2}$ Frankfurt Zoo, Bernhard-Grzimek-Allee 1, 60316 Frankfurt, Germany \\ ${ }^{3}$ Institut für Pharmakologie und Toxikologie, Justus-Liebig-Universität Gießen, Schubertstraße 81, 35392 Gießen, Germany \\ ${ }^{4}$ Institut für Lebensmittelqualität und-sicherheit, Stiftung Tierärztliche Hochschule Hannover, Bischofsholer Damm 15, \\ 30173 Hannover, Germany
}

Correspondence should be addressed to Christoph Lämmler; christoph.laemmler@vetmed.uni-giessen.de

Received 8 January 2014; Accepted 14 April 2014; Published 30 April 2014

Academic Editor: Daniel A. Feeney

Copyright (c) 2014 Karin Risse et al. This is an open access article distributed under the Creative Commons Attribution License, which permits unrestricted use, distribution, and reproduction in any medium, provided the original work is properly cited.

\begin{abstract}
The present study was designed to characterize phenotypically and genotypically an Arcanobacterium pluranimalium strain (A. pluranimalium 4868) following necropsy from a juvenile giraffe. The species identity could be confirmed by phenotypical investigations and by MALDI-TOF MS analysis, by sequencing the 16S rDNA, pluranimaliumlysin encoding gene pla, and glyceraldehyde-3-phosphate dehydrogenase encoding gene gap with sequence similarities to A. pluranimalium reference strain DSM $13483^{\mathrm{T}}$ of $99.2 \%, 89.9 \%$, and $99.1 \%$, respectively. To our knowledge, the present study is the first phenotypic and genotypic characterization of an A. pluranimalium strain isolated from a giraffe.
\end{abstract}

\section{Introduction}

Genus Arcanobacterium was described by Collins et al. 1982 [1] as a group of facultative anaerobic, asporogenous, and Gram-stain positive rods. According to Yassin et al. (2011) [2], this genus consists of four species, namely, Arcanobacterium haemolyticum, Arcanobacterium hippocoleae, Arcanobacterium phocae, and Arcanobacterium pluranimalium. More recently, Arcanobacterium canis and Arcanobacterium phocisimile, two species which were most closely related to $A$. haemolyticum, were described as novel species of this genus $[3,4]$.

The original species characterization of A. pluranimalium was performed with two strains isolated from a dead harbour porpoise and a dead fallow deer [5]. In the following years A. pluranimalium could also be isolated from a dog with pyoderma [6], from ovine specimens on 33 occasions, and from a milk sample of a single cow with mastitis [7]. More recently several $A$. pluranimalium strains recovered from various specimens were identified phenotypically and by using various molecular targets [8].

\section{Material and Methods}

The present study was focused on the characterization of an A. pluranimalium strain following necropsy from a juvenile giraffe by various phenotypic properties, by MALDI-TOF MS analysis, and genotypically by sequencing $16 \mathrm{~S}$ rDNA and the A. pluranimalium-specific target genes pla and gap.

The $80.5 \mathrm{~kg}$ female giraffe (Giraffa camelopardalis reticulata) of the present study was born in 2013. The giraffe was not accepted by its mother or wet nurse and did not 
accept hand rearing attempts and, because of general weakness, was euthanized three days after birth. The subsequent postmortem analysis revealed an acute hyperemia of lung and liver and a focal emphysema of the lung. The acute pneumonia was caused by a bacterial infection associated with aspirated foreign bodies.

Bacteriological investigations yielded the isolation of $A$. pluranimalium and Escherichia coli, partly together with coagulase negative staphylococci, $\alpha$-haemolytic streptococci, and Pseudomonas fluorescens from liver, spleen, kidney, and lung. A moderate to high growth of $E$. coli was generally noted $(++,+++)$; A. pluranimalium grew only in low numbers (+). The A. pluranimalium strain 4868, originally obtained from the spleen, was used for further studies. The bacterial strain was investigated phenotypically and by MALDI-TOF analysis [6,9] and genotypically by amplification and sequencing of $16 \mathrm{~S}$ rDNA using universal oligonucleotide primer 16 UNI-L ( $5^{\prime}$-AGA-GTT-TGA-TCATGG-CTC-AG-3) and 16 UNI-R (5'-GTG-TGA-CGG-GCGGTG-TGT-AC-3) for amplification, under the following PCR conditions: $\left(\times 1\left(95^{\circ} \mathrm{C}, 600 \mathrm{sec}\right), \times 30\left(95^{\circ} \mathrm{C}, 30 \mathrm{sec}, 58^{\circ} \mathrm{C}\right.\right.$, $60 \mathrm{sec}, 72^{\circ} \mathrm{C}, 60 \mathrm{sec}$ ), and using oligonucleotide primer 533 F (5'-GTG-CCA-GCM-GCC-GCG-GTA-A $\left.{ }^{\prime}-3\right)$ and 907R ( $5^{\prime}$-CCG-TCA-ATT-CMT-TTG-AGT-TT- ${ }^{\prime}$ ) for sequencing. The strain was also characterized by amplification of the target gene pla with the oligonucleotide primer pla-F: $5^{\prime}$ GTT GAT CTA CCA GGA TTG ACG C-3' and pla-R: $5^{\prime}$-TTG TCG GGG TGT CCA TTG CC-3' and gene gap with the oligonucleotide primer gap-F $5^{\prime}$-TTG ACC GAC AAC AAG ACC CT- $3^{\prime}$ and gap-R $5^{\prime}$-CCA TTC GTT GTC GTA CCA AG-3' as described [8, 10]. Alignment studies were performed using DNASTAR Lasergene Version 8.0.2 (DNASTAR Inc., Madison, WI, USA), Clustal W method. For MALDI-TOF MS the isolates were prepared using the direct smear method as well as an extraction protocol provided by the manufacturer. Briefly, freshly grown bacteria were harvested and diluted in ethanol, centrifuged $(2000 \times \mathrm{g})$, air-dried, and resuspended in aqueous volumes of $70 \%$ formic acid and acetonitril followed by a vortex step. Five microliters was directly transferred to the steel target. Analysis was performed on a MALDI-TOF MS Biotyper Version V3.3.1.0. The database used (DB 4613, Bruker Daltonics) comprised 45 spectra from A. pluranimalium DSM $13483^{\mathrm{T}}$.

\section{Results and Discussions}

A. pluranimalium 4868 investigated in the present study was identified by determination of hemolysis and CAMPlike hemolytic reactions, by using a commercial identification system as well as various other phenotypical tests. The CAMP-like hemolytic reactions with Staphylococcus aureus $\beta$-hemolysin, Rhodococcus equi, and Arcanobacterium haemolyticum as indicator strains are known as typicalcharacteristics of this species $[6,8,11]$. Comparable to previously investigated A.pluranimalium $[6,8]$ the phenotypical tests also revealed the typical biochemical properties of this species (Table 1). It was of interest that A. pluranimalium 4868 of the present study was catalase negative. This was observed previously for A. pluranimalium of bovine origin [8].

As shown by numerous authors MALDI-TOF MS is a powerful tool for species identification of a broad spectrum of bacteria including Gram-positive and Gram-negative bacteria [12-14]. Comparable to the previously conducted MALDITOF MS analysis of bacteria of genera Arcanobacterium and Trueperella (formerly belonging to genus Arcanobacterium $[9,15])$, MALDI-TOF MS allowed the identification of $A$. pluranimalium 4868 of the present study to the species level matching to A. pluranimalium reference strain DSM $13483^{\mathrm{T}}$ with a log score value of 2.28 .

Sequencing $16 \mathrm{~S}$ rDNA, the potentially cytolytic toxin pluranimaliumlysin encoding target gene pla and the glyceraldehyde-3-phosphate dehydrogenase encoding target gene gap revealed a sequence similarity of $99.2 \%, 89.9 \%$, and $99.1 \%$ to the respective sequences of $A$. pluranimalium DSM $13483^{\mathrm{T}}$. All three sequences of $A$. pluranimalium 4868 were deposited in GenBank (HG794511, HG423389, and HG423390). A typical dendrogram of the sequencing results of the genes pla and gap is shown in Figures 1 and 2. Comparable to gene plo of $T$. pyogenes, which appeared to be a constant characteristic of all investigated $T$. pyogenes [16-19], pla of A. pluranimalium seems to be also constantly present in all strains of this species and could be used, as described previously [8], and in the present study for molecular identification of A. pluranimalium. More recently, Moser et al. 2013 [20] also described pla as novel target for molecular identification of this species.

Sequencing of gene gap had already been described for molecular identification of staphylococcal species [21] and more recently for identification of an A. haemolyticum strain isolated from a donkey [10]. In the present study gene gap could also be used as novel target for identification of $A$. pluranimalium. Further studies will give information about the constant presence and sequence similarities of both target genes pla and gap, respectively.

\section{Conclusion}

The clinical importance of $A$. pluranimalium of the present study, which was isolated from various organs of the giraffe together with in high number appearing $E$. coli, remains unclear. Since, beside aspiration pneumonia, no other pathological findings could be detected, this might represent the route of infection. However, the isolation of this bacterial species from giraffe and the hitherto described origin harbor porpoise, fallow deer, dog, sheep, and cow emphasizes the species name $A$. pluranimalium. 
TABLE 1: Biochemical properties of A. pluranimalium 4868 investigated in the present study and A. pluranimalium DSM $13483^{\mathrm{T}}$.

\begin{tabular}{|c|c|c|}
\hline Biochemical properties & A. pluranimalium 4868 & A. pluranimalium DSM $13483^{\mathrm{T} * *}$ \\
\hline Hemolysis on sheep blood agar & + & + \\
\hline \multicolumn{3}{|l|}{ CAMP-like reaction with:* } \\
\hline Staphylococcus aureus $\beta$-hemolysin & + & + \\
\hline Streptococcus agalactiae & - & - \\
\hline Rhodococcus equi & + & + \\
\hline Arcanobacterium haemolyticum & + & + \\
\hline Reverse CAMP reaction & - & - \\
\hline Nitrate reduction & $-^{1}$ & $-{ }^{1}$ \\
\hline Pyrazinamidase & $+{ }^{1}$ & $+{ }^{1}$ \\
\hline Pyrrolidonyl arylamidase & $+{ }^{1}$ & $++^{1,2}$ \\
\hline Alkaline phosphatase & $-{ }^{1}$ & $-{ }^{1,2}$ \\
\hline$\beta$-Glucuronidase $(\beta$-GUR) & $+^{1,2,3}$ & $t^{1,2,3}$ \\
\hline$\beta$-Galactosidase $(\beta$-GAL) & $-{ }^{1},(+)^{3}$ & $--^{1},(+)^{3}$ \\
\hline$\alpha$-Glucosidase $(\alpha$-GLU) & $-{ }^{1,2,3}$ & $-1,2,3$ \\
\hline$\beta$-Glucosidase ( $\beta$-GLU) & $+^{2}$ & $t^{2}$ \\
\hline $\mathrm{N}$-Acetyl- $\beta$-glucosaminidase $(\beta$-NAG) & $-1,3$ & $-1,3$ \\
\hline Esculin ( $\beta$-glucosidase $)$ & $(+)^{1}$ & $+{ }^{1}$ \\
\hline Urease & $-{ }^{1}$ & $-{ }^{1}$ \\
\hline Gelatine & $+{ }^{1}$ & $+{ }^{1}$ \\
\hline \multicolumn{3}{|l|}{ Fermentation of: } \\
\hline Glucose & $+^{1}$ & $+^{1}$ \\
\hline Ribose & $+{ }^{1}$ & $+{ }^{1}$ \\
\hline Xylose & $(+)^{1}$ & $-{ }^{1}$ \\
\hline Mannitol & $-^{1}$ & $-{ }^{1}$ \\
\hline Maltose & $-{ }^{1}$ & $(+)^{1}$ \\
\hline Lactose & $-^{1}$ & $-{ }^{1}$ \\
\hline Saccharose & $-^{1}$ & $-{ }^{1}$ \\
\hline Glycogen & $-{ }^{1}$ & $-{ }^{1}$ \\
\hline$\alpha$-Mannosidase & $--^{2}$ & $t^{2}$ \\
\hline Catalase & - & + \\
\hline
\end{tabular}

The reactions are shown as follows: ${ }^{*}$ synergistic CAMP-like reaction with indicator strains; ${ }^{* *}$ results mostly obtained from Ülbegi-Mohyla et al., 2010 [6]; +: positive reaction; (+): weak positive reaction; -: negative reaction. ${ }^{1}$ Api-Coryne test system (Biomerieux, Nürtingen, Germany); ${ }^{2}$ tablets containing substrates (Rosco Diagnostica A/S, Taastrup, Denmark); ${ }^{3} 4$-methylumbelliferyl conjugated substrates (Sigma, Steinheim, Germany).

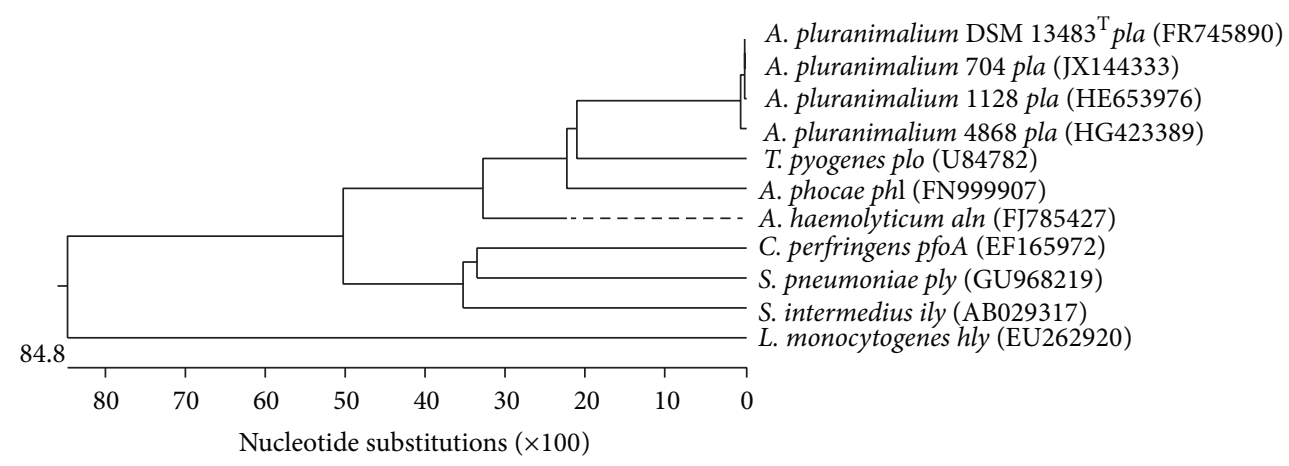

FIGURE 1: Dendrogram of sequences of gene pla of A. pluranimalium 4868 of the present study, three additional A. pluranimalium, and various other cytolytic toxin encoding genes obtained from GenBank. 


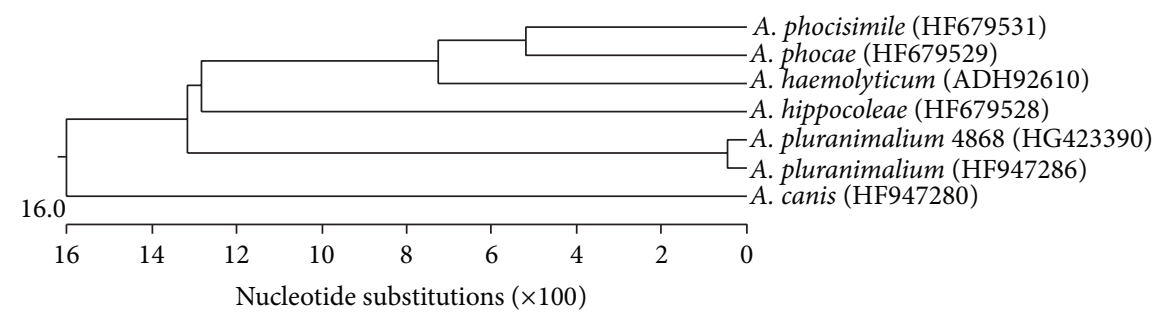

FIGURE 2: Dendrogram of gene gap of A. pluranimalium 4868, reference strain A. pluranimalium DSM $13483^{\mathrm{T}}$, and various other species of genus Arcanobacterium obtained from GenBank.

\section{Conflict of Interests}

The authors declare that they have no competing interests. The authors certify that they have no affiliation with or financial involvement in any organization or entity with a direct financial interest in the subject matter or materials discussed in this paper.

\section{References}

[1] M. D. Collins, D. Jones, and G. M. Schofield, "Reclassification of "Corynebacterium haemolyticum" (MacLean, Liebow \& Rosenberg) in the genus Arcanobacterium gen.nov. as Arcanobacterium haemolyticum nom.rev., comb.nov," Journal of General Microbiology, vol. 128, no. 6, pp. 1279-1281, 1982.

[2] A. F. Yassin, H. Hupfer, C. Siering, and P. Schumann, "Comparative chemotaxonomic and phylogenetic studies on the genus Arcanobacterium Collins et al. 1982 emend. Lehnen et al. 2006: proposal for Trueperella gen. nov. and emended description of the genus Arcanobacterium," International Journal of Systematic and Evolutionary Microbiology, vol. 61, no. 6, pp. 1265-1274, 2011.

[3] M. Hijazin, E. Prenger-Berninghoff, O. Sammra et al., "Arcanobacterium canis sp. nov., isolated from otitis externa of a dog, and emended description of the genus Arcanobacterium Collins et al. 1983 emend. Yassin et al. 2011," International Journal of Systematic and Evolutionary Microbiology, vol. 62, pp. 2201-2205, 2012.

[4] M. Hijazin, O. Sammra, H. Ülbegi-Mohyla et al., "Arcanobacterium phocisimile sp. nov., isolated from harbour seals," International Journal of Systematic and Evolutionary Microbiology, vol. 63, pp. 2019-2024, 2013.

[5] P. A. Lawson, E. Falsen, G. Foster, E. Eriksson, N. Weiss, and M. D. Collins, "Arcanobacterium pluranimalium sp. nov., isolated from porpoise and deer," International Journal of Systematic and Evolutionary Microbiology, vol. 51, no. 1, pp. 55-59, 2001.

[6] H. Ülbegi-Mohyla, A. A. Hassan, J. Alber et al., "Identification of Arcanobacterium pluranimalium isolated from a dog by phenotypic properties and by PCR mediated characterization of various molecular targets," Veterinary Microbiology, vol. 142, no. 3-4, pp. 458-460, 2010.

[7] G. Foster and B. Hunt, "Distribution of Arcanobacterium pluranimalium in animals examined in veterinary laboratories in the United Kingdom," Journal of Veterinary Diagnostic Investigation, vol. 23, no. 5, pp. 962-964, 2011.

[8] A. Balbutskaya, O. Sammra, S. Nagib et al., "Identification of Arcanobacterium pluranimalium by matrix-assisted laser desorption ionization-time of flight mass spectrometry and, as novel target, by sequencing pluranimaliumlysin encoding gene pla," Veterinary Microbiology, vol. 168, no. 2-4, pp. 428-431, 2014.

[9] M. Hijazin, A. A. Hassan, J. Alber et al., "Evaluation of matrix-assisted laser desorption ionization-time of flight mass spectrometry (MALDI-TOF MS) for species identification of bacteria of genera Arcanobacterium and Trueperella," Veterinary Microbiology, vol. 157, no. 1-2, pp. 243-245, 2012.

[10] O. Sammra, A. Balbutskaya, S. Nagib et al., "Properties of an Arcanobacterium haemolyticum strain isolated from a donkey," Berliner und Münchener Tierärztliche Wochenschrift, vol. 127, no. 1-2, pp. 10-14, 2014.

[11] H. Ülbegi-Mohyla, A. A. Hassan, T. Kanbar et al., "Synergistic and antagonistic hemolytic activities of bacteria of genus Arcanobacterium and CAMP-like hemolysis of Arcanobacterium phocae and Arcanobacterium haemolyticum with Psychrobacter phenylpyruvicus," Research in Veterinary Science, vol. 87, no. 2, pp. 186-188, 2009.

[12] P. Seng, M. Drancourt, F. Gouriet et al., "Ongoing revolution in bacteriology: routine identification of bacteria by matrixassisted laser desorption ionization time-of-flight mass spectrometry," Clinical Infectious Diseases, vol. 49, no. 4, pp. 543-551, 2009.

[13] P. R. Murray, "Matrix-assisted laser desorption ionization timeof-flight mass spectrometry: usefulness for taxonomy and epidemiology," Clinical Microbiology and Infection, vol. 16, no. 11, pp. 1626-1630, 2010.

[14] A. Bizzini, K. Jaton, D. Romo, J. Bille, G. Prod'hom, and G. Greub, "Matrix-assisted laser desorption ionization-time of flight mass spectrometry as an alternative to $16 \mathrm{~S}$ rRNA gene sequencing for identification of difficult-to-identify bacterial strains," Journal of Clinical Microbiology, vol. 49, no. 2, pp. 693696, 2011.

[15] M. Hijazin, H. Ülbegi-Mohyla, J. Alber et al., "Identification of Arcanobacterium (Trueperella) abortisuis, a novel species of veterinary importance, by matrix-assisted laser desorption ionization-time of flight mass spectrometry (MALDI-TOF MS)," Berliner und Münchener tierärztliche Wochenschrift, vol. 125, no. 1-2, pp. 32-37, 2012.

[16] S. J. Billington, B. H. Jost, W. A. Cuevas, K. R. Bright, and J. G. Songer, "The Arcanobacterium (Actinomyces) pyogenes hemolysin, pyolysin, is a novel member of the thiol-activated cytolysin family," Journal of Bacteriology, vol. 179, no. 19, pp. 6100-6106, 1997.

[17] H. B. Ertaş, A. Kiliç, G. Özbey, and A. Muz, "Isolation of Arcanobacterium (Actinomyces) pyogenes from abscessed cattle kidney and identification by PCR,' Turkish Journal of Veterinary \& Animal Sciences, vol. 29, no. 2, pp. 455-459, 2005. 
[18] H. Ülbegi-Mohyla, M. Hijazin, J. Alber et al., "Identification of Arcanobacterium pyogenes isolated by post mortem examinations of a bearded dragon and a gecko by phenotypic and genotypic properties," Journal of Veterinary Science, vol. 11, no. 3, pp. 265-267, 2010.

[19] M. Hijazin, H. Ülbegi-Mohyla, J. Alber et al., "Molecular identification and further characterization of Arcanobacterium pyogenes isolated from bovine mastitis and from various other origins," Journal of Dairy Science, vol. 94, no. 4, pp. 1813-1819, 2011.

[20] A. Moser, R. Stephan, J. Sager, S. Corti, and A. Lehner, "Arcanobacterium pluranimalium leading to a bovine mastitis: species identification by a newly developed pla gene based PCR," Schweizer Archiv für Tierheilkunde, vol. 155, no. 6, pp. 373-375, 2013.

[21] J. Yugueros, A. Temprano, B. Berzal et al., "Glyceraldehyde-3phosphate dehydrogenase-encoding gene as a useful taxonomic tool for Staphylococcus spp," Journal of Clinical Microbiology, vol. 38, no. 12, pp. 4351-4355, 2000. 

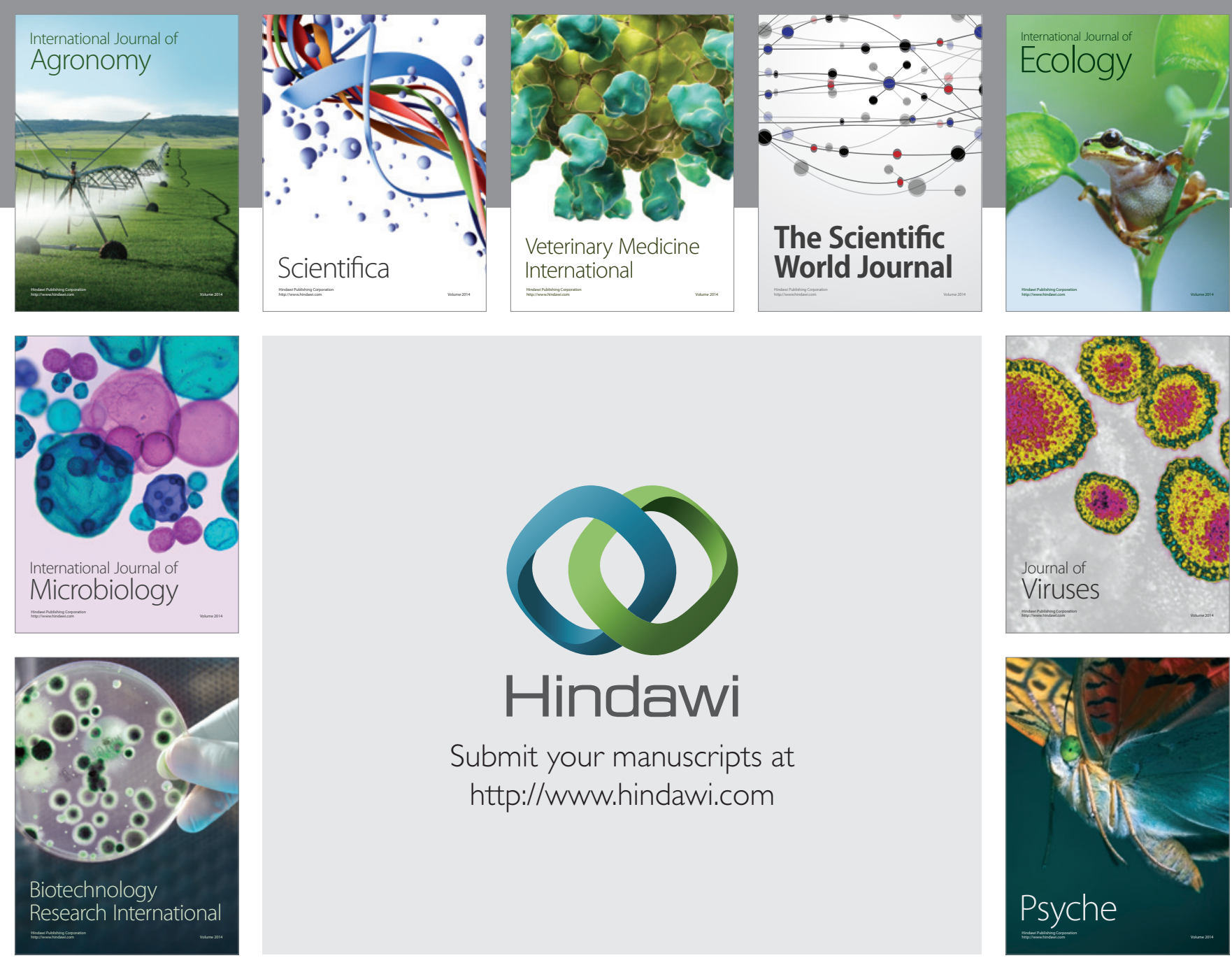

Submit your manuscripts at http://www.hindawi.com
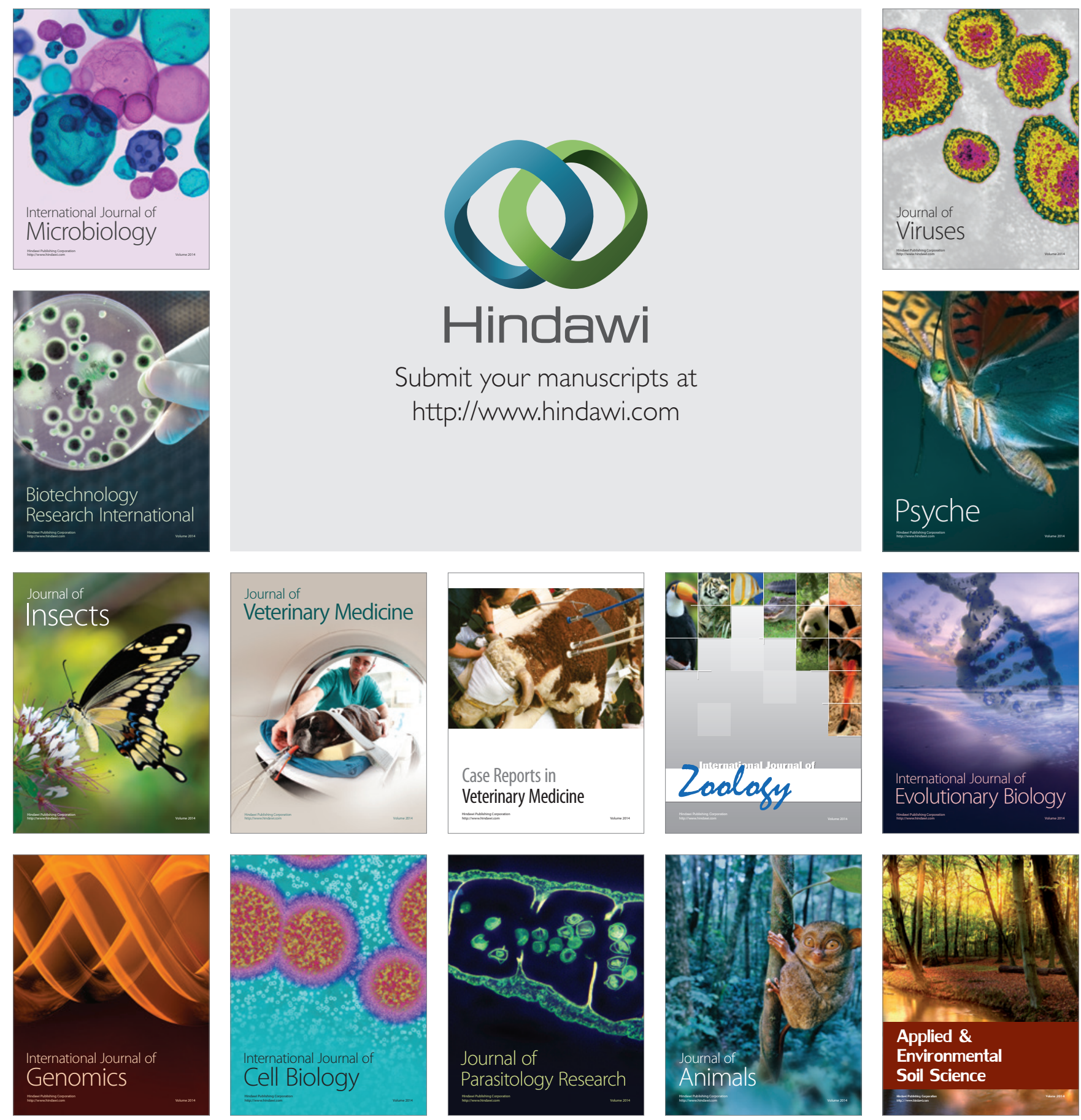